\title{
EDICT syndrome
}

INSERM

\section{Source}

INSERM. (1999). Orphanet: an online rare disease and orphan drug data base. EDICT syndrome. ORPHA:293936

EDICT (endothelial dystrophy-iris hypoplasia-congenital cataract-stromal thinning) syndrome is a very rare eye disorder representing a constellation of autosomal dominantly inherited ocular findings, including early-onset or congenital cataracts, corneal stromal thinning, early-onset keratoconus, corneal endothelial dystrophy, and iris hypoplasia. 